\title{
A technique for the identification and analysis of icebergs in Synthetic Aperture Radar images of Antarctica.
}

\begin{abstract}
This paper describes an image analysis technique developed to identify icebergs depicted in synthetic aperture radar images of Antarctica and to determine the outlines of these icebergs. The technique uses a pixel bonding process to delineate the edges of the icebergs. It then separates them from the background water and sea ice by an edge-guided image segmentation process. Characteristics such as centroid position and iceberg area are calculated for each iceberg segment and placed in a file for input to appropriate statistical data analysis software. The technique has been tested on three ERS-1 SAR sub-images in which it succeeded in identifying virtually all segments containing icebergs of size 6 pixels or larger. The images were first passed through an averaging filter to reduce speckle. This process produced a pixel size of $100 \mathrm{~m}$ x $100 \mathrm{~m}$. As implemented, the technique overestimates iceberg areas by about $20 \%$ on average and the detection rate falls off rapidly for icebergs less than six pixels in size. Performance in these areas is expected to improve when additional stages, based on a more detailed analysis of pixel intensity, are implemented.
\end{abstract}

\section{R. N. WILLIAMS* (corresponding author)}

School of Computing, University of Tasmania, PO Box 1214, Launceston

Tasmania, Australia, 7250.

Phone: + 61 (3) 63243480

Fax: $\quad+61(3) 63243368$

Email: R.Williams@utas.edu.au

* visiting scholar at the Scott Polar Research Institute (June-Sept 1996), jointly affiliated with the Cooperative Research Centre for Antarctica and the Southern Ocean and the Department of Computer Science, University of Tasmania (Oct-Dec 1996).

\section{W. G. REES}

Scott Polar Research Institute, Lensfield Road, Cambridge, CB2 1ER

United Kingdom 
and N. W. YOUNG

Cooperative Research Centre for Antarctica and the Southern Ocean,

and Australian Antarctic Division, GPO Box 252-80, Hobart, Tasmania, Australia, 7001 


\section{Introduction}

There are estimated to be of the order of 200,000 icebergs in the Southern Ocean south of the Antarctic Convergence, with linear dimensions from $50 \mathrm{~m}$ up to kilometres, and in some cases many tens of kilometres (Orheim, 1988). These icebergs form by calving from the outer margins of the Antarctic ice sheet, principally from floating ice shelves and glacier tongues, and by the fracturing of larger icebergs into two or more smaller icebergs. They are dispersed by the ocean currents. They decay by progressive fracturing, by melting, and by erosion of their sides under the action of waves. Observations of their numbers, size, linear dimensions, and spatial distribution allow their breakage and melt rates to be determined (Hamley and Budd 1986). A general overview of the physical characteristics of Antarctic icebergs is given by Kristensen (1983).

Most of the ice discharged from the Antarctic ice sheet is lost by calving of icebergs from the seaward edge of the ice sheet. Most of the remainder of the mass loss occurs by melting from the basal surfaces, especially from the floating ice in contact with the ocean. Studies of icebergs, their production and their dissolution rates provide important information on the fracture and melt processes acting on the floating margins of the ice sheet. The icebergs act as analogues of the ice shelves and glacier tongues. Their large range of sizes allows the effect of scale on the processes to be investigated. As they drift with the ocean currents, they are carried into water bodies of different temperature. They are subject to greater melt rates as well as different fracture and erosion rates in response to warmer water temperatures and different sea state. Thus, their dissolution rate in these different bodies of water provides an indication of the impact on the margins of the ice sheet of a change in oceanic conditions, which may be caused by a climatic warming, removal of the sea ice cover, or an alteration in the ocean circulation pattern.

The draft of Antarctic icebergs with lengths of a few hundred metres or greater is typically in the range from $200 \mathrm{~m}$ to $300 \mathrm{~m}$. Most of their bulk is below the water surface so that 
they move predominantly with the ocean currents and are relatively unaffected by surface winds. Thus measuring iceberg drift can be a useful method of studying the mean ocean currents for the upper few hundred metres of the ocean in a region where there are few instrumental records.

Satellite-borne synthetic aperture radar (SAR) systems provide an effective means to make observations of icebergs in polar regions. The active sensor allows images to be acquired independently of solar illumination of the surface and, because clouds are essentially transparent to the radar signals, the images are acquired irrespective of weather conditions. Icebergs can be detected in SAR images (Willis et al. 1996; Haykin et al. 1994). Analysis of the large numbers of SAR images is required for a large scale study of iceberg distribution and drift but such an analysis would only be feasible with the application of an automated or semi-automated image analysis technique for detecting the outlines of icebergs and extracting their dimensions by spatial analysis of these outlines. Such a scheme would allow the collection of data for a statistically significant population of icebergs from many different areas. It would also allow the observation of the synoptic distribution of icebergs over a large sector of the ocean and the tracking of individual icebergs in a sequence of many SAR images. The application of a set of objective rules in a self-consistent fashion would also overcome inconsistency in data arising from variations in the results produced by different manual interpretations. With the launch of the European Remote Sensing satellites (ERS-1 and ERS-2), the Japanese Earth Resources Satellite (JERS-1), and more recently the Canadian satellite, Radarsat, SAR images suitable for Antarctic applications have become available.

Although iceberg detection from remotely sensed images has received attention from relatively few researchers (Willis et al. 1996), considerable effort has been made in the area of sea ice observation using synthetic aperture radar (Vesecky et al. 1988, Carsey, 1992). Images derived from satellite-borne SAR systems have sufficiently high resolution to 
enable individual sea ice floes to be seen. Techniques have been developed (Sephton et al. 1994) which involve segmenting SAR images to produce distinct, homogeneous regions corresponding to ice and water from which various attributes such as area, mean backscatter value and shape can be determined. Thus it seemed likely that some of these techniques may be successfully used as a basis for the development of similar techniques for iceberg detection and analysis.

We describe a technique for iceberg detection under Antarctic conditions, which we have developed by adapting an ice floe segmentation method described in Sephton et al (1994). The technique has been tested on three SAR images acquired by the ERS-1 satellite during the winter month of August 1993. The images were provided as Precision Image Products (ERS.SAR.PRI) by the European Space Agency through their Announcement of Opportunity programs for the ERS satellites. The image data represent the intensity of the radar signal received at the satellite after correction for geometric and radiometric effects. Scaling and calibration factors are included with the image data to allow calculation of the backscatter coefficient. The technique we present here depends primarily on the texture properties of the images and the contrast between different elements in the images so that absolute calibration of the data is not essential for the application of the technique. The availability of calibrated data does allow criteria, such as threshold level, used in the process for distinguishing icebergs, to be assigned absolute values.

\section{Iceberg detection in synthetic aperture radar images.}

Figure 1 shows part of an ERS-1 synthetic aperture radar image (scene centre $67.86^{\circ} \mathrm{S}$ $79.74^{\circ}$ E) depicting icebergs in Prydz Bay adjacent to the Antarctic coastline, about $120 \mathrm{~km}$ north-east of Davis station. It is typical of the images acquired in East Antarctic waters that are available for analysis at the Cooperative Research Centre for Antarctica and the Southern Ocean (Antarctic CRC). The icebergs show up as regions of significantly higher 
image intensity (i.e. radar backscatter coefficient) compared to the surrounding surface which is comprised of a mix of open water and sea ice in various concentrations. The mechanisms believed to be responsible for the level of the backscatter coefficient for icebergs are described by Willis et al. (1996) and Haykin et al. (1994). Young et al. (in press) describe the conditions which affect the value of the backscatter coefficient for icebergs and their surroundings. In a manual assessment of the same three SAR scenes used in this study, they found that positively identified icebergs typically had values of the backscatter coefficient in the range from $-6 \mathrm{~dB}$ to $-4 \mathrm{~dB}$ or higher, and that $99 \%$ of the background pixels had values less than $-10.5 \mathrm{~dB}$. In an analysis of a large number of SAR scenes acquired during winter months they also found that the mean backscatter from icebergs, which were imaged on several occasions, varied by $\pm 1 \mathrm{~dB}$ or more over periods of days to weeks apparently in response to changes in local meteorological conditions. Lytle et al. (1997) describe the range of backscatter conditions for the different sea ice types found in the East Antarctic Seasonal Sea Ice Zone [SSIZ]. They found values of the backscatter coefficient in the range from $-16.5 \mathrm{~dB}$ to $-9.8 \mathrm{~dB}$ dependent on the mix of sea ice types and open water and prevailing meteorological conditions. They noted that the backscatter coefficient could be as high as $-7 \mathrm{~dB}$ with a high concentration of multi-year sea ice floes but that this was rare in the East Antarctic SSIZ. Thus, for typical conditions within the SSIZ during winter months, icebergs appear as bright targets within a darker background of pack ice and exhibit a contrast in backscatter coefficient of about $+5 \mathrm{~dB}$ or more with respect to the mean background.

Icebergs need not always exhibit such a positive contrast compared to the background of the image. On the open ocean, or within the SSIZ, wind roughened open water can have a very high backscatter which is dependent on the orientation of the wind direction relative to the look direction of the radar. The average backscatter of sea ice increases as it is broken into smaller floes. Icebergs exhibit the strongest backscatter when their surface is composed of cold dry coarse-grained snow, which is typical of conditions close to the Antarctic coast during the cold winter months. Mean annual temperature from 
meteorological observations at occupied stations around the East Antarctic coastline is around $-10^{\circ} \mathrm{C}$, and temperature extremes rarely reach melting point except in the short summer (e.g. Russell-Head and Simmonds 1993). Young and Hyland (1997) did find that the backscatter from very large icebergs could be depressed by $10 \mathrm{~dB}$ or more when snow moisture content increases significantly in conjunction with strong melt events. In East Antarctica melt events are short-lived, confined to the brief summer, and of limited spatial extent. In West Antarctica, particularly in the Antarctic Peninsular, the melt season is considerably longer although still confined to the summer months (Young and Hyland 1998). In addition, Young and Hyland (1998) did not find any significant depression of the radar backscatter coefficient for winter months in a time series of measurements over Antarctica derived from the ERS wind scatterometer instrument which uses the same frequency as the ERS SAR system. With increasing distance away from the coastline toward the northern margin of the SSIZ temperatures will be more moderate, so that it is probable that snow moisture content would increase to the point where depression of the backscatter could become noticeable. While icebergs retain their snow cover they will generally be bright targets. However, if they have negligible snow cover, either because they had little cover when they calved or because they have recently rolled over and lost the cover, they may appear as dark objects against a brighter background or with negligible or time-varying contrast compared with their surroundings.

In earlier work on detection of icebergs in the SAR scene shown in Figure 1 (McCracken, 1995) a simple image intensity threshold was used to separate the icebergs from the surrounding open water and sea ice. For that image the process was effective in identifying the objects that were icebergs but, where several icebergs were in close proximity with each other, it was unable to distinguish the individual icebergs in the group. In order to achieve useful statistics of iceberg dimensions, it is important that an analysis system can distinguish individual icebergs in a cluster in a consistent fashion in at least a majority of cases. There are two important products which can be obtained from an iceberg identification and measurement system: an assessment of total iceberg area present in an 
image and the dimensions of individual icebergs within the population. It is desirable, but not essential, that the dimensions be determined for all icebergs in the population contained within an image. The essential quality of the process is that any sample of icebergs whose dimensions are measured is representative of the population and that any tendency for biases or errors occurring in the measurements are known.

For a successful automated iceberg detection and analysis algorithm to be constructed, it is necessary to develop an automated segmentation process able to separate icebergs from the background and also able to separate any icebergs which are close to each other. Visual inspection of several ERS-1 SAR images showed that adjacent icebergs could usually be distinguished by the presence of a boundary line of pixels having slightly lower backscatter coefficient than the pixels within the icebergs (Figure 2). This may be due to the presence of unresolved areas of open water or sea ice between the icebergs. Irrespective of its cause, this reduction in backscatter coefficient provides a basis for a detection technique capable of distinguishing icebergs from the background and, at the same time, separating clusters of icebergs into their component bergs.

We present a technique, adapted from one described by Sephton et al. (1994) for segmenting ice floes from SAR images, which performs both functions. It uses homogeneity information across the image, combined with a subsequent pixel bonding process, to precisely locate edges representing iceberg boundaries, irrespective of whether the edge represents a boundary between an iceberg and the background or the boundary between two adjacent icebergs.

\section{Image preprocessing.}

The SAR images used in this study were generated by the European Space Agency (ESA) as SAR Precision Image products (ERS.SAR.PRI). They are 3-look, ground-range images 
that have been corrected for geometric and instrumental factors and for which absolute calibration parameters are provided to enable the derivation of values of radar cross-section coefficient. The image data have a pixel size of $12.5 \mathrm{~m}$ by $12.5 \mathrm{~m}$ and have a potentially high spatial resolution, nominally $30 \mathrm{~m}$, but the high level of speckle inherent in 3-look imagery limits the size of the objects that can be resolved using the radiometric information. In order to reduce this speckle to a level which would not compromise subsequent processing, the images were smoothed by an 8 x 8 pixel block-averaging process, giving a pixel size of $100 \mathrm{~m}$ by $100 \mathrm{~m}$ and an effective number of looks of 30 . This significantly reduced the speckle of the mean signal level for homogeneous areas but at the expense of degrading the spatial resolution and so limiting the potential of the technique for detecting small icebergs.

The iceberg analysis system includes modules to read an ERS-1 SAR image and display the image on the screen. An in-built zoom facility can be used to define a smaller rectangular region to which the processing can be confined. This allows the user to restrict the processing to particular areas of interest, greatly reducing analysis time when large areas of the image are not of interest and avoiding confusing results in areas where other structures may be mistaken for icebergs. The system also allows both the full image and the specific region under consideration to be displayed simultaneously. All subsequent operations are undertaken on the specific region only.

\section{Segmentation by Pixel Bonding.}

\subsection{Location of heterogeneous regions.}

The process of precisely defining the edge of the icebergs begins with determining which parts of the image exhibit significant variation and which are essentially homogeneous. The detection of heterogeneous regions can be carried out by scanning the image with an 
edge detection operator. A wide variety of such operators could be used to do this. However, a simple statistically-based edge detector, the sigma-on-mu filter (Sephton et al. 1994) has proven effective for this work. This filter is sensitive to the presence of an edge irrespective of its orientation in the image.

The detector scans the image using a 3 by 3 pixel window and calculates the standard deviation $(\sigma)$ and mean $(\mu)$ of the intensity values of the nine pixels within the window. A new image is generated from the values of $\sigma / \mu$ corresponding to each pixel. For images containing multiplicative noise, such as SAR images, the expectation value of this ratio for a homogeneous region is independent of mean intensity. However, when the window passes over the boundary between two regions with differing mean intensity, the variation in pixel value is greatly increased, thus increasing the value of $\sigma / \mu$. The sigma-on-mu filter used by Sephton et al. (1994) for ice floe segmentation was based on a 5 by 5 pixel window. However the problem of iceberg segmentation differs from that of ice floe segmentation in that the iceberg edges are sharper than those of ice floes and the contrast in backscatter between icebergs and the water / sea ice background is normally greater than the contrast in intensity between ice floes and the water background. This means that the smaller 3 by 3 pixel filter should be adequate to distinguish the more distinct edges around the icebergs, whereas it would be unlikely to produce a stable enough $\sigma / \mu$ figure for ice floe segmentation. Use of the smallest possible filter is important because we wish to detect icebergs down to only a few pixels in area. The pre-processing with a block-averaging rather than a median filter, as used by Sephton et al. (1994), will also contribute to an ability to detect icebergs that may occupy only a few pixels in an image.

The sigma-on-mu filter determines which pixels in the image are in the vicinity of an edge but does not precisely define the position of the edge. In order to identify and analyse icebergs, the system needs to define as accurately as possible the border of each iceberg. The border of an homogeneous region can be precisely defined by locating, to within one 
pixel, the line of maximum heterogeneity (i.e. maximum variation in intensity) in the boundary area between the region and the background.

The Valley-Seeking Bonding method, described in Sephton et al. (1994) and developed from a method previously described in Oddy and Rye (1983), "bonds" neighbouring pixels so that all pixels in an homogeneous region are bonded to each other, while neighbouring pixels are not bonded together if they are separated by an heterogeneous region (as determined by the sigma-on-mu filter). The important characteristic of the process is that it accurately locates (to within a pixel accuracy) the border of maximum heterogeneity between two homogeneous regions. It does this using an iterative process in which the pixels are progressively removed from the "edge" between the two homogeneous regions until the edge is precisely defined as a line made up of vertical and horizontal sections (often called crack edges ) which pass between adjacent image pixels.

To operate effectively the method needs to use an appropriate bonding threshold (T) to guide the subsequent bonding process. The size of this threshold determines the value of $\sigma / \mu$ above which a particular pixel neighbourhood would be classed as heterogeneous. It must be chosen to ensure that all iceberg borders are represented within areas classed as heterogeneous but that any small variations of backscatter level within an iceberg do not cause the iceberg to be unnecessarily broken up into a large number of smaller segments. If all icebergs were physically separated from each other, the overall outcome of the segmentation process would not be very sensitive to the choice of this threshold. However, one of the reasons for using the Valley-Seeking Bonding method is its potential to separate adjacent icebergs from each other. It does this by using subtle variations in intensity to indicate that what seems to be a single iceberg is in fact two icebergs which need to be separated. Because of this, the threshold must be chosen carefully so that it is able to distinguish subtle boundaries between two adjacent icebergs but is not confused by irrelevant minor intensity variations across the iceberg surface itself or by residual speckle in the image. 
An optimal value of $\mathrm{T}$ can be chosen by inspecting the histogram of the sigma-on-mu image. The histogram typically exhibits a prominent peak with a long tail towards the high $\sigma / \mu$ end. Since the heterogeneous regions in the image are likely to be relatively small in area compared to the homogeneous regions, we can interpret the peak as representing $\sigma / \mu$ values for pixels in the homogeneous areas. If we look for the value of $\sigma / \mu$ at which the peak (representing mainly homogeneous pixels) merges with the long tail (which represents mainly heterogeneous pixels) this should give us an estimate of the most appropriate value for T. Inspection of the $\sigma / \mu$ histograms, for the three test ERS-1 images investigated, suggested that the optimum value of $\mathrm{T}$ for these cases is somewhere within the range 0.15 to 0.20 . For subsequent evaluation work a value of 0.18 was used. In the final iceberg analysis system this value will be set as a default value, with an option for the user to vary the value interactively.

\subsection{Pixel bonding.}

Once the bonding threshold (T) has been set, the Valley-Seeking Bonding method passes a 3 by 3 pixel window across each pixel in the sigma-on-mu image. If the central pixel $\sigma / \mu$ is below T, this pixel is bonded to those of its four nearest neighbours which also have $\sigma / \mu$ values below $\mathrm{T}$. If the central pixel $\sigma / \mu$ is greater than or equal to $\mathrm{T}$, then the central pixel will be bonded to that pixel, of the four nearest neighbours, which has the minimum value of $\sigma / \mu$.

The first condition bonds together all pixels that lie in a single homogeneous region but leaves pixels in the heterogeneous (edge) regions unbonded. The second condition bonds each pixel located in an edge region with a single neighbouring pixel, either in the same edge region or in an adjacent homogeneous region, choosing the neighbouring pixel which has the lowest $\sigma / \mu$ value. The result is that the edge region between two homogeneous regions will have the pixels on one side bonded, either directly or indirectly, to pixels in the 
homogeneous region on that side and pixels on the other side bonded to pixels in the homogeneous region on that side (Figure 3). Most importantly there will be a boundary line down the interior of the edge region over which pixels will not be bonded. This boundary represents the line of maximum heterogeneity (or intensity variation) within the edge region and is a good representation of the border between the two homogeneous regions.

Once the bonds have been created, the image is scanned and all pixels which are not bonded to the pixel immediately above are labelled as having an edge above them and all those not bonded to the pixel on the right are labelled as having an edge to the right (Figure 3). Some pixels may be labelled as having an edge above and to the right. This process defines a precise edge along the line of maximum intensity variation (the true edge) but also produces a number of false edges radiating out from either side of the true edge. It is essential that these be removed in order to clearly define the true edge. These false edges are removed by iteratively scanning the image, identifying any unconnected edge ends and removing them (Sephton et al. 1994).

\subsection{Region labelling.}

Once the edges have been precisely defined the regions separated by these edges are each assigned a unique label using an adaptation of the region labelling procedure described in Sephton et al (1994). All steps of the Sephton labelling procedure have been implemented except the final one. This step involved merging some regions which had initially been given separate region labels. Instead, a straightforward region merging process, described as part of the blob-colouring algorithm in Ballard and Brown (1982), has been used in our system. The complete region labelling procedure divides the image into a relatively large number of closed regions, each uniquely labelled. The largest of these represents the background, while most of the smaller regions represent icebergs depicted in the synthetic aperture radar image. 


\section{Segment Analysis.}

\subsection{Iceberg separation and identification.}

A significant problem encountered in previous work undertaken on the problem of identification of icebergs in satellite images (McCracken 1995) has been the difficulty experienced in clearly delineating individual icebergs which are adjacent to one or more other bergs. Pairs or clusters of adjacent icebergs were identified as one iceberg, whereas the objective was to identify and analyse each iceberg separately. The Valley-Seeking Bonding technique described in this paper achieves this separation by making use of subtle decreases in image intensity which follow the boundaries of individual icebergs even where the icebergs are almost in contact with each other.

The segmentation technique is applied to the SAR images in a non-discriminatory fashion. It identifies closed regions within the image on the basis of the $\sigma / \mu$ value and independently of other properties. These closed regions may represent icebergs, sea ice or open-water, or even a mixture depending on the homogeneity of the different categories of surface and the detection threshold set for the edge identification process. Additional information, such as brightness, is required to distinguish icebergs from other segments. This is discussed further in Section 6.

\subsection{Production of iceberg statistics.}

Once the image segmentation has been completed, appropriate segment characteristics are derived and made available in tabular form. Variables considered most important in 
iceberg studies include the location of each iceberg, its area and various other shape characteristics. Currently the following variables are derived from the image segments :

(a) ID Number: this is taken to be the region label assigned during the segmentation phase, since this number is unique for each segment.

(b) Location: this is obtained by calculating the image pixel coordinates of the centroid

of each segment. These may be converted to geographical latitude and longitude by

using the navigation information that is provided in the image header.

(c) Area : this is obtained by counting the pixels in each iceberg segment and multiplying by the known area of each pixel (i.e. $100 \mathrm{~m} \times 100 \mathrm{~m}=10000$ square metres in this case).

(d) Mean backscatter coefficient: backscatter coefficient corresponding to the mean intensity averaged over all pixels in the segment.

These results are placed in a table for subsequent input to an appropriate statistical data analysis package.

\section{Performance Evaluation.}

The system described above has been developed and applied to subregions of three test images. All three images are ERS-1 synthetic aperture radar images acquired between the 6th and the 13th of August, 1993, and depict regions off the Antarctic coastline, between latitudes $67^{\circ} \mathrm{S}$ and $70^{\circ} \mathrm{S}$ and between longitudes $68^{\circ} \mathrm{E}$ and $82^{\circ} \mathrm{E}$ (Table 1). They have been 
preprocessed, as described in Section 3, by averaging blocks of pixels in the original high resolution image to create a lower resolution image, with greatly reduced speckle. One of these images was used to create the test region (Region A) shown in Figure 1. The image analysis operations outlined in Sections 4 and 5 were performed on each test region to provide a representation of the icebergs detected in that region. The result of this process for Region A is shown in Figure 4.

Evaluation of the automated analysis procedure was carried out by comparing each individual segment produced by the procedure with a manual interpretation of the corresponding area of the SAR image. The interpretation was done on the basis of spatial character and brightness of the pixels to identify which segments were icebergs and to determine the number of pixels representing each individual iceberg. The maximum daily temperature measured at Mawson and Davis stations at the time of acquisition of the SAR images was about $-15^{\circ} \mathrm{C}$. Mawson is $300 \mathrm{~km}$ to the west of Region $\mathrm{B}$, and Davis is within $120 \mathrm{~km}$ of Regions A and C and $370 \mathrm{~km}$ east of Region B. Therefore the surface of an iceberg would be completely frozen and appear as a bright object because of its high backscatter coefficient. The results of this evaluation are given in Table 2.

The 719 segments delineated in the image regions A, B and C are identified as:

(a) well-defined icebergs where the segment corresponds to a single iceberg and the shape of the segment corresponds closely with that of the iceberg,

(b) over-segmented icebergs in which the segment represents only part of an iceberg with other parts being represented by other segments,

(c) under-segmented areas containing two or more icebergs which were not resolved by the segmentation process,

(d) poorly defined icebergs where the segment represents a single iceberg but with poorly defined boundaries and containing pixels with a brightness intermediate between iceberg backscatter levels and background levels, 
(e) doubtful icebergs which could not be definitely identified manually as icebergs but which may contain sub-pixel size fragments of iceberg, and

(f) false identifications in which the segment does not coincide with any manually identified iceberg.

An additional 95 icebergs were observed manually but were not identified as separate segments by the analysis technique. These are designated in Table 2 as missed icebergs. They appeared as brighter pixels within the more homogeneous areas of the background. Most of them were only one pixel in size and only one exceeded 5 pixels, so that they represented a tiny fraction of the iceberg mass and a negligible fraction of the image area.

A simple test using a threshold level and the mean brightness of a segment was implemented in an attempt to automatically differentiate segments containing icebergs from those without. The threshold was set at the 99th percentile of the brightness values of the background for each test region. It was expected that if the mean value was less than the threshold then the pixels of the segment belonged to the background population and if greater than the threshold they represented iceberg. Less than $8 \%$ of the segments were incorrectly classified on this basis. Those segments mostly belonged to the marginal categories of poorly defined or doubtful icebergs and had mean brightness values close to the threshold. A more robust definition of the threshold and more complex testing of the pixels within a segment should resolve most if not all of these mis-classifications.

The segmenting process tended to include, within the segments representing icebergs, some proportion of pixels from the background, so that their size was over-estimated. During the manual assessment process the number of pixels representing each iceberg was counted. A comparison of the detected area with the manually assessed area shows that the automated process overestimated the total area by about $20 \%$. The proportion of over-estimation for individual icebergs varied considerably. A difference of a few pixels for small icebergs represented a large proportion. For larger iceberg sizes the differences tended to represent 
a smaller proportion of the area. Since it is an effect at the boundary of an iceberg it is expected that on average the difference would vary approximately with the length of the perimeter.

In the ideal case, the icebergs would be clearly defined within a background of homogeneous texture so that the segment boundaries would closely match the edges of the icebergs. In reality the background can be of quite heterogeneous texture so that lines of maximum heterogeneity need not coincide exactly with the iceberg boundaries. Most of these extraneous pixels can be eliminated on the basis of their intensity properties. In addition, some pixels around the margins of an iceberg will contain a sub-pixel mix of iceberg and background and have an intermediate intensity value. For an accurate estimate of the iceberg size, a portion of these pixels will need to be assigned to the iceberg. The worst estimate of size occurs with the poorly defined segments which contain a significant fraction of pixels assessed to represent the background. Further processing is required to better identify the iceberg within these segments and reject the extraneous background pixels. In a few cases, a pixel assessed manually to belong to an iceberg segment was excluded by the segmentation process and remained within the background or was assigned to one of the false identifications. Occasionally such a pixel was attached to a neighbouring iceberg segment.

For the areas that were under- or over-segmented, the actual number of icebergs is given in Table 2 in parentheses. The 30 under-segmented segments within the three image regions contained a total of 63 individual icebergs, whereas the 78 over-segmented segments actually represented only 34 icebergs. The segmentation process is sensitive to the choice of bonding threshold which needs to balance over-segmentation of a few icebergs in some areas against a failure to segment icebergs present in other areas. The over-segmenting of icebergs is not a problem if the goal is to estimate the total iceberg mass, but it is a considerable disadvantage when deriving statistics and dimensions of individual icebergs. One of the difficulties in carrying out the evaluation is that the only source of information is 
the images themselves. It is possible that the segmentation process is more effective than manual interpretation in separating what could be individual icebergs in close proximity.

A disadvantage of using the edge detection approach, as opposed to absolute intensity thresholding for iceberg detection, is that it is difficult for the edge-based technique to reliably detect icebergs with areas significantly smaller than the 3 by 3 filter used for edge detection. Therefore it is expected that the technique would decrease in reliability when attempting to identify icebergs smaller than 9 pixels in area. However, the segmenting operation found a total of 126 segments with a size less than 6 pixels and these had about the same rate of well-defined iceberg identifications and false identifications as the larger segments. The process succeeded in identifying virtually all icebergs of size 6 pixels or larger and many of those with a size of 4 or 5 pixels.

A histogram which shows the number of segments classified as containing an iceberg falling within specified ranges of size for Region A is presented in Figure 5. It is indicative of the information that is provided by this image analysis system. Segments have been omitted from this sample where they were assessed to be in the poorly defined or doubtful identification categories. The areas of a total of 127 iceberg segments are presented. The area axis is on a log scale with a doubling in area occurring for each two size classes. There is one segment in the sample with an area between 4 and $5.7 \mathrm{~km}^{2}$. All other segments have an area less than $1 \mathrm{~km}^{2}$. The mode of the distribution occurs at a mean class size of $0.15 \mathrm{~km}^{2}$. The numbers of iceberg segments in the smaller size classes are underestimated compared to the manual assessment.

Young et al. (in press) have applied the technique described here in the analysis of more than 200 ERS SAR scenes. In that work they used the technique as a computer-guided analysis system to define the boundary of segments in the image and used a threshold intensity to distinguish valid iceberg segments from false identifications. They then undertook a manual verification stage where an operator either (a) confirmed the analysis 
for each iceberg, (b) combined the individual sections of an over-segmented iceberg or (c) reapplied the segmentation stage to a small area of the image with different bonding threshold to separate icebergs in an under-segmented section. This combination of the automated stages and manual intervention facilitated a rapid analysis of the images, allowing many images to be completed in a day. The extraction of area, linear dimensions, and other properties was undertaken automatically by a final spatial analysis stage after the verification stage.

They presented results from observations of over 4000 icebergs in 54 of those scenes. A qualitative assessment made as these analyses were carried out confirmed the character and magnitude of the findings presented here regarding the effectiveness of the technique and the biases currently present in the derived dimensions. They omitted from their statistics of the iceberg populations any observations that would be designated here as in the doubtful, poorly-defined or missed categories. They found that the technique identified almost all icebergs down to a size of six pixels and that the combined area of the icebergs identified represented $85-90 \%$ or more of the total area of the iceberg population in the images.

\section{Conclusions and future work.}

The work reported in this paper tested the feasibility of automatically detecting and analysing icebergs depicted in synthetic aperture radar imagery of Antarctica. A segmentation algorithm, adapted from one originally designed to outline sea ice floes in SAR images, has been applied to the iceberg detection problem. The advantage of this algorithm, compared with previous techniques used to perform iceberg detection (McCracken 1995), is its ability to identify individual icebergs even when they were closely packed together in clusters. 
The adapted algorithm has proven effective in detecting icebergs in ERS SAR images. It responded to the presence of all icebergs, greater than 6 pixels in size, by producing segments containing these icebergs. We still need to improve its ability to precisely define iceberg shape and its ability to eliminate segments which don't contain icebergs but the technique is able to produce an initial image segmentation capable of serving as an excellent basis for further analysis. Currently the segmentation process generally overestimates the area of icebergs and the technique is not reliable for icebergs less than 6 pixels in area. The detection performance should be improved and better size estimates achieved when additional stages are implemented based on the analysis of pixel intensity. On the basis of a trial processing of several Radarsat images from Antarctica, we anticipate that the performance of the technique on images from other SAR systems will be of the same order as the performance reported here for the ERS-1 test images.

Future development work with the system will be directed to improving the accuracy of iceberg outlines and area measures and to detecting smaller icebergs. It is intended to follow the existing edge-based procedure with a simple backscatter threshold procedure, based on that described by McCracken (1995), to detect the smaller icebergs. The threshold level would be set from a knowledge of the distribution of backscatter values for those icebergs which have been positively identified by the edge-based procedure. The simple thresholding process could differentiate iceberg pixels from background pixels with a greater precision than the edge-based procedure and should be able to reliably detect icebergs down to 1 pixel in area where they exhibit sufficient contrast with respect to the background.

It is also possible that the edge-based algorithm would be able to detect smaller icebergs in a higher resolution product, such as that produced by using a $4 \times 4$ pixel block in the averaging process applied to the original ERS-1 images. This would generate an image with $50 \mathrm{~m}$ by $50 \mathrm{~m}$ pixels and, although such an image would have more residual speckle in it, the improved spatial resolution may more than compensate for this, allowing the algorithm to 
detect smaller icebergs. We intend to carry out an analysis of the trade-off between spatial resolution, averaging window size and speckle reduction and will alter the initial averaging process applied to the images if this improves the overall effectiveness of the technique.

The technique has already been successfully applied to a survey of iceberg sizes near their sources on the Antarctic coast (Young et al. in press). Further studies to be made in the near future include analysis of the dissolution rates of the icebergs by fracture and melt, and the drift of icebergs with the ocean currents. For this work, we intend to apply the iceberg identification technique to the analysis of images from SAR systems on the ERS, JERS, Radarsat and future Envisat satellites.

\section{Acknowledgements.}

We wish to acknowledge the support of a research grant awarded by the Antarctic Science Advisory Committee (Project No 908) for some of this work. Ray Williams also wishes to thank all at the Scott Polar Research Institute, University of Cambridge, for making the excellent resources of the Institute available to him for the duration of his study leave and for ensuring that he spent a very enjoyable and fruitful time in Cambridge.

The ERS.SAR.PRI images were supplied by the European Space Agency through the ERS Announcement of Opportunity projects ERS.AO.AUS5-1 and ERS.AO2.AUS103 (Principal Investigator - N. Young) and are copyright to ESA, 1993.

\section{References.}

BALLARD, D.H. and BROWN, C.H. 1982, Computer Vision, Prentice-Hall, New Jersey. 
CARSEY, F.D. 1992, Microwave Remote Sensing of Sea Ice, American Geophysical Union Monograph 68, American Geophysical Union, Washington, D.C.

HAMLEY, T.C. and BUDD, W.F. 1986, Antarctic iceberg distribution and dissolution. Journal of Glaciology, 32 (111), 242-251.

HAYKIN, S., LEWIS, E.O., RANEY, K.R. and ROSSITER, J.R., 1994, Remote Sensing of Sea Ice and Icebergs, John Wiley and Sons Inc., New York, N.Y.

KRISTENSEN, M., 1983, Iceberg calving and deterioration in Antarctica. Progress in Physical Geography, 7 (3), 313-328.

LYTLE, V.I., MASSOM, R., WORBY, A.P. and ALLISON, I. 1997. Floe sizes in the East Antarctic sea ice zone estimated using combined SAR and field data. In Proceedings of the Third ERS Scientific Symposium, 17-21 March 1997, Florence, Italy. Frascati, Italy, European Space Agency, 931-936. (ESA Publication SP-414.)

McCRACKEN, C., 1995, Techniques for Identifying and Classifying Icebergs in Synthetic Aperture Radar Images, Bachelor of Applied Computing Honours Thesis, Department of Applied Computing and Mathematics, University of Tasmania.

ODDY, C.J. and RYE, A.J., 1983, Segmentation of SAR images using a local similarity rule. Pattern Recognition Letters, 1, 443-449.

ORHEIM, O., 1988, Antarctic icebergs - production, distribution and disintegration. (Abstract). Annals of Glaciology, 11, 205. 
RUSSELL-HEAD, D. and SIMMONDS, I., 1993, Temporal structure of surface weather parameters at Casey, Davis, Mawson and Macquarie Island. Meteorology Section, School of Earth Sciences, The University of Melbourne, Publication No. 35, Pp. 181.

SEPHTON, A.J., BROWN, L.M.J, MACKLIN, J.T., PARTINGTON, K.C., VECK, N.J. and REES, W.G., 1994, Segmentation of Synthetic-Aperture Radar imagery of sea ice. International Journal of Remote Sensing, 15, 803-825.

VESECKY. J.F., SAMADANI, R., SMITH, M.P., DAIDA, J.M. and BRACEWELL, R.N., 1988, Observation of sea-ice dynamics using Synthetic-Aperture Radar images: Automated analysis. IEEE Transactions on Geoscience and Remote Sensing, 26, 11371145.

WILliS, C.J., MACKLIN, J.T., PARTINGTON, K.C., TELEKI, K.A., REES, W.G. and WILLIAMS, R.G., 1996, Iceberg detection using ERS-1 Synthetic Aperture Radar. International Journal of Remote Sensing, 26, 1777-1795.

YOUNG, N.W. and HYLAND, G., 1997, Applications of time series of microwave backscatter over the Antarctic region. In Proceedings of the Third ERS Scientific Symposium, 17-21 March 1997, Florence, Italy. Frascati, Italy, European Space Agency, 1007-1014. (ESA Publication SP-414.)

YOUNG, N.W. and HYLAND, G., 1998, Interannual variability of Antarctic snow melt events derived from scatterometer data. In Proceedings of the 1998 IEEE International Geoscience and Remote Sensing Symposium (IGARSS'98), 6-10 July 1998, Seattle, USA. IEEE Publication., Piscataway, New Jersey, 2261-2263.

YOUNG, N.W., TURNER, D., HYLAND, G. and WILLIAMS, R.N., in press, Near coastal iceberg distributions in East Antarctica $50^{\circ} \mathrm{E}-145^{\circ} \mathrm{E}$. Annals of Glaciology, 27. 
Region.

Acquisition date

ERS-1 Orbit number

ERS-1 Frame number

Scene Centre Latitude

Scene Centre Longitude
A $\quad$ B $\quad$ C

$\begin{array}{rrr}\text { 06-Aug-93 } & \text { 13-Aug-93 } & \text { 06-Aug-93 } \\ 10755 & 10865 & 10755 \\ 5013 & 5787 & 5049 \\ 67.86^{\circ} \mathrm{S} & 67.64^{\circ} \mathrm{S} & 69.38^{\circ} \mathrm{S} \\ 79.74^{\circ} \mathrm{E} & 69.63^{\circ} \mathrm{E} & 77.04^{\circ} \mathrm{E}\end{array}$

Table1. Details of ERS-1 SAR images used in this work. 
Region.

Number of segments outlined

Well-defined icebergs

Over-segmented icebergs

Under-segmented areas

Poorly defined icebergs

Doubtful icebergs

False identifications

Missed icebergs
A

219

98

(11) 24

(11) 5

18

19

55

40

4
B

C

Total

98

306

192

719

$\begin{array}{lll}134 & 64 & 296\end{array}$

(14) 30

(9) 24

(34) 78

(34) 17

(18) 8

(63) 30

8

26

9

53

12

9

40

87

78

220

20

35

95

Table 2. Performance evaluation results for the technique on the three sub-regions. The number in parentheses () gives the number of icebergs manually assessed to be represented by the number of segments identified. 


\section{Figure Captions.}

Figure 1. Part of a SAR image depicting icebergs off the Antarctic coastline, between latitudes $67^{\circ} \mathrm{S}$ and $70^{\circ} \mathrm{S}$ and between longitudes $68^{\circ} \mathrm{E}$ and $82^{\circ} \mathrm{E}$. The test region shown here is referred to as Region A and covers an area $(23 \mathrm{~km}$ on a side) off Princess Elizabeth Land, approximately north east of Davis station. Pixels from the original ERS-1 SAR image were averaged in 8 by 8 pixel blocks

to produce this image, reducing speckle significantly. [ERS SAR data (C) ESA, 1993]

Figure 2. Enlarged sub-region in Region A, depicting a cluster of three adjacent icebergs

(centre of image). The contrast in this image has been enhanced to reveal the subtle boundary lines of slightly lower backscatter coefficient which allow the analysis technique to separate these icebergs. [ERS SAR data (C) ESA, 1993]

Figure 3. Pixel Bonding and Edge Location. The homogeneous pixels (white) are bonded to all neighbouring homogeneous pixels while the inhomogeneous pixels (grey) are bonded to the neighbouring pixel which has a minimum $\sigma / \mu$

value. The result of this process reveals the true edge (thick dashed line) and many false edges (thin dashed lines) because pixels will not be bonded across these lines. The false edges can be removed by an iterative process in which edge ends are progressively removed until none remain.

Figure 4. Outlines of icebergs depicted in Figure 1. These are outlines of the segments produced by the segmentation process described in Section 4. 
Figure 5. Segment size distribution produced by the system for Region A. Each vertical bar represents a specified size range, while the height of the bar represents the number of iceberg segments in Region A which fall into that size range. 
Short title (of 60 characters or less for a running headline).

Analysis of icebergs in SAR images of Antarctica. 